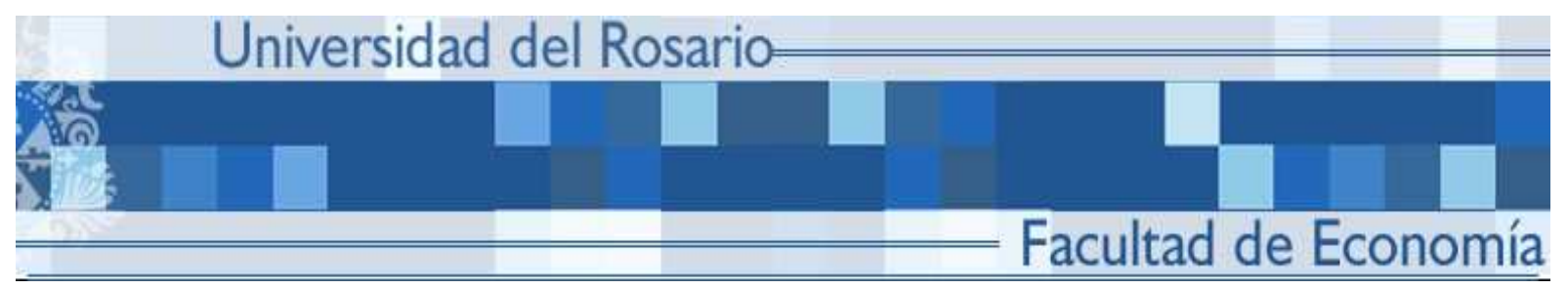

\title{
ON THE EXHAUSTIVENESS OF TRUNCATION AND DROPPING STRATEGIES IN MANY-TO-MANY MATCHING MARKETS
}

Paula Jaramillo

Çağatay Kayi

Flip Klijn

\section{SERIE DOCUMENTOS DE TRABAJO}

\author{
No. 123
}

Septiembre de 2012 


\title{
On the Exhaustiveness of Truncation and Dropping Strategies in Many-to-Many Matching Markets*
}

\author{
Paula Jaramillo ${ }^{\dagger}$ Çağatay Kayı ${ }^{\ddagger}$ and Flip Klijn ${ }^{\S}$
}

\begin{abstract}
We consider two-sided many-to-many matching markets in which each worker may work for multiple firms and each firm may hire multiple workers. We study individual and group manipulations in centralized markets that employ (pairwise) stable mechanisms and that require participants to submit rank order lists of agents on the other side of the market. We are interested in simple preference manipulations that have been reported and studied in empirical and theoretical work: truncation strategies, which are the lists obtained by removing a tail of least preferred partners from a preference list, and the more general dropping strategies, which are the lists obtained by only removing partners from a preference list (i.e., no reshuffling).

We study when truncation / dropping strategies are exhaustive for a group of agents on the same side of the market, i.e., when each match resulting from preference manipulations can be replicated or improved upon by some truncation / dropping strategies. We prove that for each stable mechanism, truncation strategies are exhaustive for each agent with quota 1 (Theorem 1). We show that this result cannot be extended neither to group manipulations (even when all quotas equal 1 - Example 1), nor to individual manipulations when the agent's quota is larger than 1 (even when all other agents' quotas equal 1 - Example 2). Finally, we prove that for each stable mechanism, dropping strategies are exhaustive for each group of agents on the same side of the market (Theorem 2), i.e., independently of the quotas.
\end{abstract}

Keywords: matching, many-to-many, stability, manipulability, truncation strategies, dropping strategies.

JEL-Numbers: C78, D60.

\footnotetext{
*We thank the seminar participants at Universidad de los Andes, Universidad del Rosario, GAMES 2012, and First Caribbean Game Theory Conference for valuable discussions.

${ }^{\dagger}$ Corresponding author. Universidad de los Andes, Facultad de Economía, Calle 19A \# 11 - 37, Bloque W, Bogotá, Colombia; e-mail: p.jaramillo26@uniandes.edu.co

${ }^{\ddagger}$ Universidad del Rosario, Bogotá, Colombia. Ç. Kayı gratefully acknowledges the hospitality of Institute for Economic Analysis (CSIC) and financial support from Colciencias/CSIC (Convocatoria No: 506/2010), El Patrimonio Autónomo Fondo Nacional de Financiamiento para la Ciencia, la Tecnología y la Innovación, Francisco José de Caldas.

${ }^{\S}$ Institute for Economic Analysis (CSIC) and Barcelona GSE, Spain. The first draft of this paper was written while F. Klijn was visiting Universidad del Rosario. He gratefully acknowledges the hospitality of Universidad del Rosario and financial support from CSIC/Colciencias 2010CO0013, Plan Nacional I+D+i (ECO2011-29847), and the Government of Catalonia (SGR2009-01142).
} 


\section{Introduction}

In part-time labor markets and some professional entry-level labor markets a worker may be employed by a number of different firms. An important example of the latter are British entrylevel medical labor markets which involve graduating medical students and teaching hospitals. Each student seeks two residency positions: one for a medical program and one for a surgical program. Roth (1991) modeled the British entry-level medical labor markets as many-to-two matching markets.

In this paper, we consider many-to-many matching markets in which each worker may work for multiple firms and each firm may hire multiple workers. Agents have preferences over subsets of potential partners. An assignment between workers and firms is called a matching. A central concept in the matching literature is (pairwise) stability. A matching is called stable if all agents are matched to an acceptable subset of partners and there is no unmatched worker-firm pair who both would prefer to match (and possibly dismiss some current partners). Roth (1984a) studied a general many-to-many model and showed that if the agents' preferences satisfy substitutability then the set of stable matchings is non-empty. ${ }^{1}$

In many-to-one matching markets, the set of stable matchings coincides with the core. In addition, ruling out blocking pairs is sufficient for ruling out blocking coalitions that involve more than two agents. This is not true in many-to-many matching markets. Not only might the set of stable matchings be different from the core, but also there might be stable matchings that can be blocked by coalitions of more than two agents. Sotomayor (1999b) studied the stronger concept of setwise stability and showed that in the many-to-many model the set of stable matchings, the core, and the set of setwise stable matchings do not coincide. However, potential larger blocking coalitions in complex real-life settings might have more difficulties to organize themselves. In fact, Roth (1991, page 422) suggested that for many-to-many markets such as the British entry-level medical labor markets, stability is still of primary importance.

Many real-life matching markets employ a centralized mechanisms to match workers with firms and the only information that the matchmaker asks from the participating agents are their preferences over the other side of the market. In particular, we assume that the agents' quotas (i.e., the number of available slots) are commonly known by the agents (because, for instance, the quotas are determined by laws). ${ }^{2}$ In practice, agents are only allowed to submit ordered lists of individual partners (potential partners that are not listed are assumed to be unacceptable). Presumably the agents' preferences over sets of potential partners are responsive (Roth, 1985a): for each agent $i$, the convenience to match with an additional potential partner $j$ by possibly replacing some partner $k$ only depends on the individual characteristics of $j$ and $k$ (and whether the quota is reached). Throughout the current paper we focus on mechanisms that only demand ordered lists of potential individual and acceptable partners and keep the

\footnotetext{
${ }^{1}$ Substitutability was introduced by Kelso and Crawford (1982) to show the existence of stable matchings in a many-to-one model with money.

${ }^{2}$ In particular, quotas cannot be manipulated (cf. Sönmez, 1997).
} 
responsiveness assumption. ${ }^{3}$ A mechanism is stable if for each reported profile of ordered lists it produces a matching that is stable with respect to the reported profile. Two important examples of such mechanisms are the so-called worker-optimal and firm-optimal stable mechanisms which are based on the deferred acceptance algorithm (introduced by Gale and Shapley, 1962, for the one-to-one case and adapted by Roth, 1984a, to the many-to-many case).

Even though there is evidence that clearinghouses that employ stable mechanisms often perform better than those that employ unstable mechanisms, ${ }^{4}$ no stable mechanism is immune to preference manipulation (Dubins and Freedman, 1981, and Roth, 1982). This fact immediately triggers a question: What types of strategies should a strategic agent consider? In the present paper, we focus on two types of "simple" preference manipulations that have been reported and studied in empirical and theoretical work. The first class of preference manipulations is that of truncation strategies (Roth and Vande Vate, 1991). A truncation strategy is a list that is obtained from an agent's true preference list by removing a tail of its least preferred potential partners. ${ }^{5}$ The second class of preference manipulations consists of dropping strategies (Kojima and Pathak, 2009). A dropping strategy is a list that is obtained from an agent's true preference list by removing potential partners (i.e., no reshuffling). Obviously, each truncation strategy is also a dropping strategy. Roth and Rothblum (1999) studied the firm-optimal stable mechanism in the many-to-one model. They showed that if a worker's incomplete information is completely symmetric, then it might only gain by reporting a truncation strategy. Ehlers (2008) obtained a similar result for all so-called priority and linear programming mechanisms. Coles (2009) constructively examined truncation strategies in the one-to-one model. He established that also in asymmetric incomplete information settings workers can truncate lists with little risk of ending up unmatched, but with the potential to see large gains. Ma (2010) studied truncation strategies and the equilibrium outcomes induced by the workers-optimal mechanism in one-toone and many-to-one matching markets. For one-to-one, he found that if in equilibrium each firm uses a truncation strategy, then the equilibrium outcome is the firms-optimal matching. For many-to-one, he found that if in equilibrium each firm uses a truncation strategy, then the equilibrium outcome is either the firms-optimal matching or an unstable matching with respect to the true preferences.

Taking the stability requirement for a mechanism to perform well as granted, we study stable mechanisms, but do not restrict ourselves to the firm-optimal stable mechanism (as in Roth and Rothblum, 1999, and Coles, 2009). On the other hand, we assume a complete information environment. Consider the point of view of an individual worker while keeping the other agents' strategies fixed. The worker's match induced by any strategy (i.e., reported list) of this worker can be replicated or improved upon by some truncation strategy in one-to-one markets (Roth

\footnotetext{
${ }^{3}$ Responsiveness implies substitutability, and hence the existence of a stable matching. Moreover, the set of stable matchings does not depend on the agents' particular responsive extensions.

${ }^{4}$ See, for instance, Roth (1991).

${ }^{5}$ Truncation strategies have been observed in practice. See, for instance, the empirical study of sororities in Mongell and Roth, 1991.
} 
and Vande Vate, 1991, Theorem 2). ${ }^{6}$ In other words, the worker would not lose strategic "opportunities" by focusing only on the set of truncation strategies. In view of our analysis it is convenient to restate this result as follows. Let the truncation / dropping correspondence be the correspondence that assigns to each preference relation the set of truncation / dropping strategies obtained from the induced list over individual agents. Then, in one-to-one markets, the truncation correspondence is exhaustive (Roth and Vande Vate, 1991, Theorem 2) in the sense that for each strategy, the induced match can be replicated or improved upon by some truncation of the list induced by the agent's true preference relation. However, since Roth and Vande Vate's (1991) model is one-to-one, their result would not apply to most real-life matching markets. ${ }^{7}$ We extend Roth and Vande Vate's (1991) result by showing that for each stable mechanism, the truncation correspondence is exhaustive for each agent with quota 1 (Theorem 1).

We also study when the truncation / dropping correspondence is exhaustive for a group of agents on the same side of the market, i.e., when the induced match resulting from joint preference manipulations can be replicated or improved upon by some truncation / dropping strategies. We complement our first main result with two examples to show that it cannot be generalized in the following two ways. The truncation correspondence is

- neither necessarily exhaustive for a group of agents on the same side of the market if for all $i \in I, q_{i}=1$ (Example 1);

- nor necessarily exhaustive for agent $a$ if $q_{a}>1$ and for all $i \in I \backslash\{a\}, q_{i}=1$ (Example 2).

Next, in view of Examples 1 and 2, we turn our attention to the set of dropping strategies, which includes the set of truncation strategies. Kojima and Pathak (2009, Lemma 1) proved that the dropping correspondence is exhaustive for a firm in the many-to-one model (where workers' quotas equal one). ${ }^{8}$ However, their result does not say anything about possible joint manipulations of a group of workers or a group of firms, nor deals with the possibility of workers having a larger quota than one. ${ }^{9}$ We show that for each stable mechanism, the dropping correspondence is exhaustive for each group of agents on the same side of the market (Theorem 2).

To put our paper in perspective, we briefly mention some of the most closely related papers on many-to-many matching markets (apart from the already mentioned work by Roth, 1984a,

\footnotetext{
${ }^{6}$ Roth and Vande Vate (1991) studied random stable mechanisms. We rephrase their Theorem 2 to fit it for our framework.

${ }^{7}$ For each many-to-one market, there is a one-to-one correspondence between its stable matchings and those of a related one-to-one market. Hence, many properties of the set of stable matchings in the one-to-one model carry over to the many-to-one model. Yet, with respect to strategic issues, Roth (1985a) showed that the two models are not equivalent.

${ }^{8}$ In fact, Kojima and Pathak (2009) also considered strategic manipulation by underreporting quotas. We focus on manipulation via preference lists, and aim to establish "exhaustiveness results" (of truncation and dropping strategies) for different classes of quota vectors.

${ }^{9}$ Note that we only consider (pairwise) stability, and hence do not allow for larger blocking coalitions than worker-firm pairs. This is not a conceptual contradiction to our study of joint manipulations, since larger blocking coalitions would involve agents from both sides of the market, while the joint manipulations we study only deal with groups of agents from the same side of the market.
} 
and Sotomayor, 1999b). Alkan (1999,2001,2002), Baïou and Balinski (2000), Blair (1988), Fleiner (2003), Roth (1985b), and Sotomayor (1999a) provided important insights into the lattice structure of the set of stable matchings in different (many-to-many) models. Martínez et al. (2004) presented an algorithm to compute the full set of stable matchings when preferences are substitutable. Sotomayor (2004) provided a mechanism that implements the set of stable matchings when preferences are responsive. Klijn and Yazıcı (2011) studied the number and the set of filled slots in stable matchings when preferences are substitutable and weakly separable. Finally, Echenique and Oviedo (2006), Klaus and Walzl (2009), Konishi and Ünver (2006), and Sotomayor (1999b) analyzed the relation between various solution concepts different from (pairwise) stability on several domains of preferences.

The remainder of the paper is organized as follows. In Section 2, we introduce the model. In Section 3, we present and prove our results.

\section{Model}

There are two finite and disjoint sets of agents: a set of workers $\boldsymbol{W}$ and a set of firms $\boldsymbol{F}$. Let $\boldsymbol{I}=W \cup F$ be the set of agents. We denote a generic worker, firm, and agent by $w, f$, and $i$, respectively. For each agent $i$, there is an integer quota $\boldsymbol{q}_{\boldsymbol{i}} \geq 1$. Worker $w$ can work for at most $q_{w}$ firms and firm $f$ can hire at most $q_{f}$ workers. Let $q=\left(q_{i}\right)_{i \in I}$.

Let $i \in I$. The set of potential partners of agent $i$ is denoted by $\boldsymbol{N}_{\boldsymbol{i}}$. If $i \in W, N_{i}=F$ and if $i \in F, N_{i}=W$. A subset of potential partners $N \subseteq N_{i}$ is feasible (for agent $i$ ) if $|N| \leq q_{i}$. Let $\mathcal{N}\left(N_{i}, q_{i}\right)=\left\{N \subseteq N_{i}:|N| \leq q_{i}\right\}$ denote the collection of feasible subsets of potential partners. The element $\varnothing \in \mathcal{N}\left(N_{i}, q_{i}\right)$ denotes "being unmatched" or some outside option. Agent $i$ has a complete, transitive, and strict preference relation $>_{\boldsymbol{i}}$ over $\mathcal{N}\left(N_{i}, q_{i}\right)$. For each $N, N^{\prime} \in \mathcal{N}\left(N_{i}, q_{i}\right)$, we write $N \geq_{i} N^{\prime}$ if agent $i$ finds $N$ at least as good as $N^{\prime}$, i.e., $N>_{i} N^{\prime}$ or $N=N^{\prime}$. Let $\mathcal{P}_{i}^{>}$be the set of all preference relations for agent $i$. Let $>=\left(>_{i}\right)_{i \in I}$. For $A \subseteq I$, let $>_{A}=\left(>_{i}\right)_{i \in A}$ and $>_{-A}=\left(>_{i}\right)_{i \in I \backslash A}$.

Let $\boldsymbol{P}_{\boldsymbol{i}}$ be the restriction of $>_{i}$ to $\left\{\{j\}: j \in N_{i}\right\} \cup\{\varnothing\}$, i.e., individual partners in $N_{i}$ and being unmatched. For $j, j^{\prime} \in N_{i} \cup\{\varnothing\}$, we write $j P_{i} j^{\prime}$ if $j>_{i} j^{\prime}$, and $j R_{i} j^{\prime}$ if $j \geq_{i} j^{\prime}{ }^{10}$ Let $\mathcal{P}_{i}$ be the set of all such restrictions for agent $i$. Agent $j \in N_{i}$ is an acceptable partner for agent $i$ if $j P_{i} \varnothing$. Let $P=\left(P_{i}\right)_{i \in I}$. For $A \subseteq I$, let $P_{A}=\left(P_{i}\right)_{i \in A}$ and $P_{-A}=\left(P_{i}\right)_{i \in I \backslash A}$.

We also represent an agent $i$ 's preferences $P_{i}$ as an ordered list of the elements in $N_{i} \cup\{\varnothing\}$. For instance, $P_{w}=f_{3} f_{2} \varnothing f_{1} \ldots f_{4}$ indicates that $w$ prefers $f_{3}$ to $f_{2}, f_{2}$ to being unmatched, and being unmatched to any other firm.

We assume that for each agent $i,>_{i}$ is a responsive extension of $P_{i}$ (or responsive for short), ${ }^{11}$ i.e., for all $N \in \mathcal{N}\left(N_{i}, q_{i}\right)$,

(r1) if $j \in N_{i} \backslash N$ and $|N|<q_{i}$, then $N \cup j>_{i} N$ if and only if $j P_{i} \varnothing$; and

(r2) if $j \in N_{i} \backslash N$ and $k \in N$, then $(N \backslash k) \cup j>_{i} N$ if and only if $j P_{i} k$.

\footnotetext{
${ }^{10}$ With some abuse of notation we often write $x$ for a singleton $\{x\}$.

${ }^{11}$ See Roth (1985a) and Roth and Sotomayor (1989) for a discussion of this assumption.
} 
A (many-to-many matching) market is given by $(W, F,>, q)$ or, when no confusion is possible, $(>, q)$ for short. ${ }^{12}$

Let $(W, F,>, q)$ be a market. A matching is a function $\mu: I \rightarrow 2^{I}$ such that

(m1) for all $i \in I, \mu(i) \in \mathcal{N}\left(N_{i}, q_{i}\right)$; and

(m2) for all $w \in W$ and $f \in F, f \in \mu(w)$ if and only if $w \in \mu(f)$.

Let $\mu$ be a matching. Let $i, j \in I$. If $j \in \mu(i)$ then we say that $i$ and $j$ are matched to one another and that they are mates in $\mu$. The set $\mu(i)$ is agent $i$ 's match.

Next, we describe desirable properties of matchings. First, we are interested in a voluntary participation condition over the matchings. Formally, a matching $\mu$ is individually rational if for each $i \in I$ and each $j \in \mu(i), j P_{i} \varnothing .^{13}$

Second, we aim to avoid particular blocking pairs that would render a matching unstable. Formally, a worker-firm pair $(w, f)$ is a blocking pair for $\mu$ if

(b1) $w \notin \mu(f)$;

(b2) $\left[|\mu(w)|<q_{w}\right.$ and $\left.f P_{w} \varnothing\right]$ or [ there is $f^{\prime} \in \mu(w)$ such that $\left.f P_{w} f^{\prime}\right]$; and

(b3) $\left[|\mu(f)|<q_{f}\right.$ and $\left.w P_{f} \varnothing\right]$ or $\left[\right.$ there is $w^{\prime} \in \mu(f)$ such that $\left.w P_{f} w^{\prime}\right] .^{14}$

A matching is (pairwise) stable if it is individually rational and there are no blocking pairs. Let $S(>, q)$ be the set of stable matchings for market $(>, q)$. Roth (1984a) showed that the set of stable matchings is always non-empty. In fact, he showed that for each market $(>, q)$, there is a (worker-optimal) stable matching $\mu^{W}$ that is weakly preferred by all workers to any other stable matching in $S(>, q)$. Formally, for each $w \in W$ and each $\mu \in S(>, q), \mu^{W}(w) \geq_{w} \mu(w)$. Similarly, there is a (firm-optimal) stable matching $\mu^{F}$ that is weakly preferred by all firms to any other stable matching in $S(>, q)$. Note that stability does not depend on the particular responsive extensions of the agents' preferences over individual acceptable partners. ${ }^{15}$ Hence, we can denote the set of stable matchings for $(>, q)$ by $S(P, q)$.

In many-to-one matching markets, the set of stable matchings coincides with the core. In addition, ruling out blocking pairs is sufficient for ruling out blocking coalitions that involve more than two agents. This is not true in many-to-many matching markets. Not only might the set of stable matchings be different from the core, but also there might be stable matchings that can be blocked by coalitions of more than two agents (see Sotomayor, 1999b). However, Roth (1991, page 422) suggested that for certain many-to-many markets, stability is still of primary importance.

A mechanism assigns a matching to each market. We assume that quotas are commonly

\footnotetext{
${ }^{12} \mathrm{~A}$ many-to-one matching market is a market where each agent on one given side of the market has quota 1. A one-to-one or marriage market is a market where each agent has quota 1.

${ }^{13}$ Alternatively, by responsiveness condition (r1), a matching $\mu$ is individually rational if no agent would be better off by breaking a match, i.e., for each $i \in I$ and each $j \in \mu(i), \mu(i)>_{i} \mu(i) \backslash j$.

${ }^{14}$ By responsiveness conditions (r1) and (r2), (b2) is equivalent to $\left[\left[|\mu(w)|<q_{w}\right.\right.$ and $\left.\mu(w) \cup f>_{w} \mu(w)\right]$ or [there is $f^{\prime} \in \mu(w)$ such that $\left.\left(\mu(w) \backslash f^{\prime}\right) \cup f>_{w} \mu(w)\right]$ ]. A similar equivalent statement holds for (b3).

${ }^{15}$ In fact, the set of stable matchings does not depend either on the agents' orderings of the (individual) unacceptable partners.
} 
known by the agents (because, for instance, the quotas are determined by law). ${ }^{16}$ Therefore, the only information that the mechanism asks from the agents are their preferences over the other side of the market. ${ }^{17}$ Many real-life centralized matching markets employ mechanisms that only ask for the ordered lists $P=\left(P_{i}\right)_{i \in I}$ of individual partners, i.e., they do not depend on the particular responsive extensions. Throughout the paper we focus on this class of mechanisms. Hence, a mechanism $\varphi$ assigns a matching $\varphi(P, q)$ to each pair $(P, q)$. We often denote agent $i$ 's match $\varphi(P, q)(i)$ by $\varphi_{i}(P, q)$. A mechanism $\varphi$ is stable if for each $(P, q), \varphi(P, q) \in S(P, q)$. Two important examples of such mechanisms are the worker-optimal stable mechanism $\varphi^{W}$ and the firm-optimal stable mechanism $\varphi^{F}$ which assign to each market its worker-optimal stable matching and firm-optimal stable matching, respectively.

An important question is whether stable mechanisms are immune to preference manipulations by strategic agents. A strategy is an (ordered) preference list of a subset of potential partners. ${ }^{18}$ More precisely, for each agent $i, \mathcal{P}_{i}$ is the set of strategies. Dubins and Freedman (1981) and Roth (1982) showed that there is no stable mechanism that is strategy-proof. Formally, for each stable mechanism, $\varphi$, there is a market $(>, q)$ in which some agent $i$ can submit a preference list $P_{i}^{\prime}$ different from its true preference list $P_{i}$ and obtain a better match, i.e., $\varphi_{i}\left(P_{i}^{\prime}, P_{-i}, q\right)>_{i} \varphi_{i}(P, q)$.

Next, we provide the formal definition of two important classes of strategies that have been studied in the literature. A truncation strategy of a worker $w$ is an ordered list $P_{w}^{\prime}$ obtained from $P_{w}$ by making a tail of acceptable firms unacceptable (Roth and Vande Vate, 1991). Formally, for a worker $w$ with preferences $P_{w}$ over individual firms, $P_{w}^{\prime}$ is a truncation strategy if for any firms $f, f^{\prime} \in F$, (a) [if $f R_{w}^{\prime} f^{\prime} R_{w}^{\prime} \varnothing$ then $f R_{w} f^{\prime} R_{w} \varnothing$ ], and (b) [if $f P_{w}^{\prime} \varnothing$ and $f^{\prime} P_{w} f$ then $\left.f^{\prime} P_{w}^{\prime} \varnothing\right]$. We define a truncation strategy of a firm similarly.

A dropping strategy of a worker $w$ is an ordered list $P_{w}^{\prime}$ obtained from $P_{w}$ by removing some acceptable firms, i.e., not necessarily a tail of least preferred firms (Kojima and Pathak, 2009). Formally, for a worker $w$ with preferences $P_{w}$ over individual firms, $P_{w}^{\prime}$ is a dropping strategy if for any firms $f, f^{\prime} \in F,\left[f R_{w}^{\prime} f^{\prime} R_{w}^{\prime} \varnothing\right.$ implies $\left.f R_{w} f^{\prime} R_{w} \varnothing\right]$. We define a dropping strategy of a firm similarly.

A strategy space reductor for $i$ is a correspondence $\boldsymbol{\Sigma}$ that maps each preference relation $>_{i}$ to a subset of the set of strategies. Formally, a strategy space reductor is a correspondence $\Sigma: \mathcal{P}_{i}^{>} \rightrightarrows \mathcal{P}_{i}$ such that for each $>_{i} \in \mathcal{P}_{i}^{>}$, the (non-empty) reduced strategy space $\Sigma\left(>_{i}\right)$ is a subset of $\mathcal{P}_{i}$. We focus on two strategy space reductors: the truncation correspondence and the dropping correspondence. The truncation correspondence $\boldsymbol{\tau}$ associates each preference relation $>_{i}$ with the set of truncation strategies obtained from the corresponding restriction $P_{i}$. Similarly, the dropping correspondence $\boldsymbol{\delta}$ associates each preference relation $>_{i}$ with the set of dropping strategies obtained from the corresponding restriction $P_{i}$.

We next define the exhaustiveness of a strategy space reductor for an individual agent, i.e.,

\footnotetext{
${ }^{16}$ In particular, quotas cannot be manipulated (cf. Sönmez, 1997).

${ }^{17}$ Nonetheless, we do not suppress the notation $q$ since the quotas play a role in the definition of stability. Moreover, our results are also conditional on the values of the quotas.

${ }^{18}$ The listed potential partners are interpreted as the acceptable potential partners. The other potential partners are unacceptable and, since we focus on stable mechanisms, their relative ordering is irrelevant.
} 
when a strategy space reductor is rich enough to replicate or improve upon any possible match. Let $q$ be a quota vector, $\varphi$ be a mechanism and $\Sigma$ be a strategy space reductor. The strategy space reductor $\Sigma$ is $\boldsymbol{\varphi}$-exhaustive for agent $\boldsymbol{i}$ if for each $>_{i}$, each $P_{i}^{\prime}$, and each $P_{-i}$, there exists $Q_{i} \in \Sigma\left(>_{i}\right)$ such that $\varphi_{i}\left(Q_{i}, P_{-i}, q\right) \geq_{i} \varphi_{i}\left(P_{i}^{\prime}, P_{-i}, q\right)$.

When groups of agents on the same side of the market can jointly carry out strategic manipulations, we extend the previous definition as follows. Let $q$ be a quota vector, $\varphi$ be a mechanism, and $A^{\prime} \subseteq A$ be a group of agents on the same side of the market $A \in\{W, F\}$. A (common) strategy space reductor $\Sigma$ is $\boldsymbol{\varphi}$-exhaustive for group $\boldsymbol{A}^{\prime}$ if for each $>_{A^{\prime}}$, each $P_{A^{\prime}}^{\prime}$, and each $P_{-A^{\prime}}$, there exists $Q_{A^{\prime}} \in \times_{i \in A^{\prime}} \Sigma\left(>_{i}\right)$ such that for each $i \in A^{\prime}, \varphi_{i}\left(Q_{A^{\prime}}, P_{-A^{\prime}}, q\right) \geq_{i} \varphi_{i}\left(P_{A^{\prime}}^{\prime}, P_{-A^{\prime}}, q\right)$.

Note that $\varphi$-exhaustiveness for a group of agents implies $\varphi$-exhaustiveness for an agent, but the reverse is not true (see, for instance, Theorem 1 and Example 1).

\section{Results}

In this section, we present and prove our results. We first consider the truncation correspondence and seek to determine when it is exhaustive. Recall that the quotas $\left(q_{i}\right)_{i \in I}$ are fixed and cannot be manipulated.

Roth and Vande Vate (1991) studied a matching model making the following assumptions: (1) $|W|=|F|$, (2) each agent is acceptable to all agents on the other side of the market, and (3) for each $i \in I, q_{i}=1$. Their Theorem 2 says that for each stable mechanism $\varphi$, the truncation correspondence $\tau$ is $\varphi$-exhaustive for each agent. It can easily be seen that the first two assumptions can be disposed of. Below, we further extend the result by relaxing the third assumption as well. Note that in the proof we conveniently appeal to the exhaustiveness of the dropping correspondence (see Theorem 2).

Theorem 1. Let $A \in\{W, F\}$. Let $\varphi$ be a stable mechanism. Suppose for some $a \in A, q_{a}=1$. Then, the truncation correspondence $\tau$ is $\varphi$-exhaustive for agent a.

Proof. Let $\varphi$ be a stable mechanism. Let $(>, q)$ be a market. Let $P$ be the restriction of $>$ to individual partners and being unmatched. Without loss of generality, let $A=W$. Let $w \in W$ be such that $q_{w}=1$.

Let $P_{w}^{\prime}$ be a strategy for $w$. We identify a truncation strategy $Q_{w} \in \tau\left(>_{w}\right)$ with $\varphi_{w}\left(Q_{w}, P_{-w}, q\right)$ $R_{w} \varphi_{w}\left(P_{w}^{\prime}, P_{-w}, q\right)$. By Theorem 2 , there is a dropping strategy $P_{w}^{*}$ with $\varphi_{w}\left(P_{w}^{*}, P_{-w}, q\right) R_{w}$ $\varphi_{w}\left(P_{w}^{\prime}, P_{-w}, q\right)$. Then, it is enough to identify a truncation strategy $Q_{w}$ with $\varphi_{w}\left(Q_{w}, P_{-w}, q\right)$ $R_{w} \varphi_{w}\left(P_{w}^{*}, P_{-w}, q\right)$. We distinguish between two cases.

Case I. $\varnothing R_{w} \varphi_{w}\left(P_{w}^{*}, P_{-w}, q\right)$.

Let $Q_{w}=\varnothing$ be the empty truncation strategy. Then, by the stability of $\varphi, \varphi_{w}\left(Q_{w}, P_{-w}, q\right)=\varnothing$. Hence, $\varphi_{w}\left(Q_{w}, P_{-w}, q\right) R_{w} \varphi_{w}\left(P_{w}^{*}, P_{-w}, q\right)$.

Case II. $\varphi_{w}\left(P_{w}^{*}, P_{-w}, q\right) P_{w} \varnothing$.

Note that $\varphi_{w}\left(P_{w}^{*}, P_{-w}, q\right) \in F$. Let $f^{*}=\varphi_{w}\left(P_{w}^{*}, P_{-w}, q\right)$. Let $Q_{w}$ be the truncation of $P_{w}$ such that $f^{*}$ is the last acceptable firm. Let $Q=\left(Q_{w}, P_{-w}\right)$. We first show that for all $\mu \in S(Q, q)$, 
$\mu(w) R_{w} f^{*}$.

Suppose, to the contrary, that there is some $\tilde{\mu} \in S(Q, q)$ with $f^{*} P_{w} \tilde{\mu}(w)$. Then, since each firm $f$ with $f^{*} P_{w} f$ is not listed (i.e., acceptable) in $Q_{w}$ and since $\tilde{\mu}$ is individually rational with respect to $Q, \tilde{\mu}(w)=\varnothing$. By Roth $(1984 \mathrm{~b}$, Theorem 9$)$, for all $\mu \in S(Q, q), \mu(w)=\varnothing$. In particular, $\varphi_{w}^{W}(Q, q)=\varnothing$.

Note that $\varphi^{W}(Q, q)$ is stable under $\left(P_{w}^{*}, P_{-w}, q\right)$. Suppose, to the contrary, that there is a blocking pair for $\varphi^{W}(Q, q)$ under $\left(P_{w}^{*}, P_{-w}, q\right)$. Then, the same pair blocks $\varphi^{W}(Q, q)$ under $(Q, q)$. Hence, $\varphi^{W}(Q, q)$ is not stable under $(Q, q)$, contradicting the stability of $\varphi^{W}$. Since $\varphi_{w}^{W}(Q, q)=\varnothing$, by Alkan (2002, Proposition 6) and the stability of $\varphi, \varphi_{w}\left(P_{w}^{*}, P_{-w}, q\right)=\varnothing$, contradicting $\varphi_{w}\left(P_{w}^{*}, P_{-w}, q\right)=f^{*}{ }^{19}$ Hence, for all $\mu \in S(Q, q), \mu(w) R_{w} f^{*}$. Since $\varphi(Q, q) \in$ $S(Q, q), \varphi_{w}(Q, q) R_{w} f^{*}=\varphi_{w}\left(P_{w}^{*}, P_{-w}, q\right)$.

We complement Theorem 1 with two examples to show that it cannot be extended in the following two ways. The truncation correspondence is

- neither necessarily $\varphi$-exhaustive for a group of agents on the same side of the market if for all $i \in I, q_{i}=1$ (Example 1);

- nor necessarily $\varphi$-exhaustive for agent $a$ if $q_{a}>1$ and for all $i \in I \backslash\{a\}, q_{i}=1$ (Example 2).

Example 1. (The truncation correspondence $\tau$ is not necessarily $\varphi$-exhaustive for a group of agents on the same side of the market if for all $i \in I, q_{i}=1$.) Consider the one-to-one matching market $(W, F,>, q)$ with 4 workers, 4 firms, and preferences $P$ given by the columns in Table 1. Only acceptable partners are depicted in Table 1. For instance, $P_{w_{1}}=f_{4} f_{2} f_{3} \varnothing f_{1}$. For each agent $i \in I, q_{i}=1$.

\begin{tabular}{|c|c|c|c|c|c|c|c|}
\hline \multicolumn{4}{|c|}{ Workers } & \multicolumn{4}{|c|}{ Firms } \\
\hline$w_{1}$ & $w_{2}$ & $w_{3}$ & $w_{4}$ & $f_{1}$ & $f_{2}$ & $f_{3}$ & $f_{4}$ \\
\hline$f_{4}$ & $f_{1}$ & $f_{3}$ & $f_{4}$ & $w_{3}$ & $w_{4}$ & $w_{1}$ & $w_{2}$ \\
\hline$f_{2}$ & \begin{tabular}{|l|}
$f_{4}$ \\
\end{tabular} & $f_{1}$ & $f_{3}$ & $w_{4}$ & $w_{1}$ & $w_{4}$ & $w_{1}$ \\
\hline$f_{3}$ & & & $f_{1}$ & $w_{2}$ & & $w_{3}$ & $w_{4}$ \\
\hline & & & $\begin{array}{l}f_{2} \\
\end{array}$ & & & & \\
\hline
\end{tabular}

Table 1: Preferences $P$ in Example 1

One easily verifies that the firm-optimal stable matching $\mu=\varphi^{F}(P, q)$ is given by

\footnotetext{
${ }^{19}$ Proposition 6 in Alkan (2002) is an extension of part of a result that is known as the Rural Hospital Theorem (Roth, 1984b).
} 


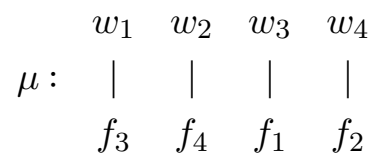

which is the boxed matching in Table 1.

Consider the profile of (dropping) strategies $\left(P_{w_{1}}^{\prime}, P_{w_{2}}^{\prime}\right)$ where $P_{w_{1}}^{\prime}=f_{2}$ and $P_{w_{2}}^{\prime}=f_{1}$. Let $P^{\prime}=\left(P_{w_{1}}^{\prime}, P_{w_{2}}^{\prime}, P_{-\left\{w_{1}, w_{2}\right\}}\right)$. The firm-optimal stable matching $\mu^{\prime}=\varphi^{F}\left(P^{\prime}, q\right)$ now equals

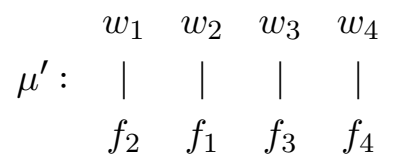

which is the boldfaced matching in Table 1 . Note that $\mu^{\prime}\left(w_{1}\right)=f_{2} P_{w_{1}} f_{3}=\mu\left(w_{1}\right)$ and $\mu^{\prime}\left(w_{2}\right)=$ $f_{1} P_{w_{2}} f_{4}=\mu\left(w_{2}\right)$. It follows that under the firm-optimal stable mechanism, in market $(>, q)$ workers $\left\{w_{1}, w_{2}\right\}$ can strictly improve their matches by jointly misreporting their preferences.

\begin{tabular}{l|l|c}
$Q_{w_{1}}$ & $Q_{w_{2}}$ & $\varphi_{w_{2}}^{F}\left(Q_{w_{1}}, Q_{w_{2}} P_{-\left\{w_{1}, w_{2}\right\}}\right)$ \\
\hline$f_{4} f_{2} f_{3}$ & $f_{1} f_{4}$ & $f_{4}$ \\
$f_{4} f_{2}$ & $f_{1} f_{4}$ & $f_{4}$ \\
$f_{4}$ & $f_{1} f_{4}$ & $f_{4}$ \\
$f_{4} f_{2} f_{3}$ & $f_{1}$ & $\varnothing$ \\
$f_{4} f_{2}$ & $f_{1}$ & $\varnothing$ \\
$f_{4}$ & $f_{1}$ & $\varnothing$
\end{tabular}

Table 2: Truncations of $w_{1}, w_{2}$ and matches of $w_{2}$ in Example 1

In Table 2, we indicate the match of worker $w_{2}$ under the firm-optimal stable mechanism $\varphi^{F}$ for each profile $\left(Q_{w_{1}}, Q_{w_{2}} P_{-\left\{w_{1}, w_{2}\right\}}\right)$ where $Q_{w_{1}}$ and $Q_{w_{2}}$ are truncation strategies. One immediately verifies that no pair of truncation strategies for $w_{1}$ and $w_{2}$ leads to a match for $w_{2}$ that is weakly preferred to $f_{1}=\varphi_{w_{2}}^{F}\left(P_{w_{1}}^{\prime}, P_{w_{2}}^{\prime}, P_{-\left\{w_{1}, w_{2}\right\}}\right)$.

Example 2. (The truncation correspondence $\tau$ is not necessarily $\varphi$-exhaustive for agent $\boldsymbol{a} \in \boldsymbol{A}$ if $\boldsymbol{q}_{\boldsymbol{a}}>1$ and for all $\left.\boldsymbol{i} \in \boldsymbol{I} \backslash \boldsymbol{a}, \boldsymbol{q}_{\boldsymbol{i}}=\mathbf{1 .}\right)^{20,21}$ Consider a many-to-one matching market $(W, F,>, q)$ with 3 workers, 4 firms, and preferences over individual partners $P$ given by the columns in Table 3. All potential partners are acceptable. For instance, $P_{f_{1}}=w_{3} w_{1} w_{2} \varnothing$. Worker $w_{1}$ has quota $q_{w_{1}}=2$. Any other agent $i$ has quota $q_{i}=1$.

One easily verifies that the unique stable matching $\mu$ for $(P, q)$ is given by

$$
\mu: \begin{array}{ccc}
w_{1} & w_{2} & w_{3} \\
\left\{f_{3}, f_{4}\right\} & f_{2} & f_{1}
\end{array}
$$

\footnotetext{
${ }^{20}$ The preferences $P$ are adapted from Roth (1985a, p. 283, Table I) and Roth and Sotomayor (1990, p. 146).

${ }^{21}$ By introducing additional workers and firms, the negative result in Example 2 can be extended in a straightforward way to situations in which for all $i \in I \backslash\{a\}, q_{i} \geq 1$.
} 


\begin{tabular}{|c|c|c|c|c|c|c|}
\hline \multicolumn{3}{|c|}{ Workers } & \multicolumn{4}{|c|}{ Firms } \\
\hline$w_{1}$ & $w_{2}$ & $w_{3}$ & $f_{1}$ & $f_{2}$ & $f_{3}$ & $f_{4}$ \\
\hline$f_{1}$ & $f_{1}$ & $f_{3}$ & $w_{3}$ & $w_{2}$ & $w_{1}$ & $\overline{w_{1}}$ \\
\hline$f_{2}$ & $f_{2}$ & \begin{tabular}{|l|}
$f_{1}$ \\
\end{tabular} & $w_{1}$ & $w_{1}$ & $w_{3}$ & $w_{2}$ \\
\hline$f_{3}$ & $f_{3}$ & $f_{2}$ & $w_{2}$ & $w_{3}$ & $w_{2}$ & $w_{3}$ \\
\hline$f_{4}$ & $f_{4}$ & $f_{4}$ & & & & \\
\hline
\end{tabular}

Table 3: Preferences $P$ in Example 2

which is the boxed matching in Table 3 .

Consider the (dropping) strategy $P_{w_{1}}^{\prime}=f_{1} f_{4}$ for worker $w_{1}$. Let $P^{\prime}=\left(P_{w_{1}}^{\prime}, P_{-w_{1}}\right)$. The unique stable matching for $\left(P^{\prime}, q\right)$ is given by

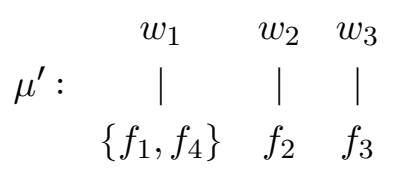

which is the boldfaced matching in Table 3. Note that $\mu^{\prime}\left(w_{1}\right)=\left\{f_{1}, f_{4}\right\}>_{w_{1}}\left\{f_{3}, f_{4}\right\}=\mu\left(w_{1}\right)$ for each responsive extension $>_{w_{1}}$ of $P_{w_{1}}$. Since $\mu$ and $\mu^{\prime}$ are the unique stable matchings for $(P, q)$ and $\left(P^{\prime}, q\right)$, respectively, it follows that under each stable mechanism, in market $(>, q)$ firm $f_{1}$ can strictly improve its match by misreporting its preferences.

\begin{tabular}{l|c}
$Q_{w_{1}}$ & $\varphi_{w_{1}}\left(Q_{w_{1}}, P_{-w_{1}}\right)$ \\
\hline$f_{1} f_{2} f_{3} f_{4}$ & $\left\{f_{3}, f_{4}\right\}$ \\
$f_{1} f_{2} f_{3}$ & $f_{3}$ \\
$f_{1} f_{2}$ & $f_{1}$ \\
$f_{1}$ & $f_{1}$
\end{tabular}

Table 4: Truncations and matches of $w_{1}$ in Example 2

In Table 4, we indicate the match of worker $w_{1}$ under each stable mechanism $\varphi$ and for each profile $\left(Q_{w_{1}}, P_{-w_{1}}\right)$ where $Q_{w_{1}}$ is a truncation strategy. One immediately verifies that no individual truncation strategy for $w_{1}$ replicates or improves upon the match $\left\{f_{1}, f_{4}\right\}=\varphi_{w_{1}}\left(P_{w_{1}}^{\prime}, P_{-w_{1}}\right)$.

As is clear from the previous examples, the truncation correspondence is not exhaustive for all possible vectors of quotas. For this reason we now turn to the set of dropping strategies, which includes the set of truncation strategies.

Kojima and Pathak (2009) considered a many-to-one matching model where for each $w \in W$, $q_{w}=1$. Their Lemma 1 implies that for each stable mechanism $\varphi$, the dropping correspondence is $\varphi$-exhaustive for each firm $f \in F$. We extend this result by showing that for each stable 
mechanism $\varphi$, the dropping correspondence is $\varphi$-exhaustive for a group of agents on the same side of the market, independently of the vector of the quotas. The proof parallels that of Kojima and Pathak (2009, Lemma 1). The main difference with their proof is that we need to show that during the procedure to get a stable matching only firms with vacant positions can be part of blocking pairs.

Theorem 2. Let $\varphi$ be a stable mechanism. The dropping correspondence $\delta$ is $\varphi$-exhaustive for a group of agents on the same side of the market.

Proof. Let $\varphi$ be a stable mechanism. Let $(>, q)$ be a market. Let $P$ be the restriction of $>$ to individual partners and being unmatched. Without loss of generality, let $A=W$. Let $W^{\prime} \subseteq W$.

Let $P_{W^{\prime}}^{\prime}=\left(P_{i}^{\prime}\right)_{i \in W^{\prime}}$ be a strategy-profile for $W^{\prime}$. Let $\mu=\varphi\left(P_{W^{\prime}}^{\prime}, P_{-W^{\prime}}, q\right)$. For each $w \in W^{\prime}$, let $I^{\mu}(w)=\left\{f: f \in \mu(w)\right.$ and $\left.f P_{w} \varnothing\right\}$ be the set of firms matched to $w$ at $\mu$ and that are acceptable for $w$ with respect to $P_{w}$. For each $w \in W^{\prime}$, let $Q_{w} \in \delta\left(>_{w}\right)$ be the dropping strategy obtained from $P_{w}$ by ranking the firms in $I^{\mu}(w)$ according to the true relative ordering and making all other firms unacceptable. We need to show that for all $w \in W^{\prime}, \varphi_{w}\left(Q_{W^{\prime}}, P_{-W^{\prime}}, q\right) \geq_{w}$ $\varphi_{w}\left(P_{W^{\prime}}^{\prime}, P_{-W^{\prime}}, q\right)$. Note that by $(\mathrm{r} 1)$ in the definition of responsiveness it is sufficient to show that for each $w \in W^{\prime}, \varphi_{w}\left(Q_{W^{\prime}}, P_{-W^{\prime}}, q\right)=I^{\mu}(w)$.

For each $w \in W$, let

$$
\mu_{0}^{\prime}(w)=\left\{\begin{array}{cc}
I^{\mu}(w) & \text { if } w \in W^{\prime} \\
\mu(w) & \text { if } w \notin W^{\prime}
\end{array}\right.
$$

Suppose $\mu_{0}^{\prime}$ is stable with respect to $\left(Q_{W^{\prime}}, P_{-W^{\prime}}, q\right)$. Let $w \in W^{\prime}$. Note that in $\mu_{0}^{\prime}$ agent $w$ is assigned to all its acceptable partners (with respect to $Q_{w}$ ). Hence, by Alkan (2002, Proposition 6), for each stable matching $\nu \in S\left(Q_{W^{\prime}}, P_{-W^{\prime}}, q\right), \nu(w)=\mu_{0}^{\prime}(w)=I^{\mu}(w)$. By stability of $\varphi, \varphi_{w}\left(Q_{W^{\prime}}, P_{-W^{\prime}}, q\right)=I^{\mu}(w)$, which we needed to establish.

Suppose $\mu_{0}^{\prime}$ is not stable with respect to $\left(Q_{W^{\prime}}, P_{-W^{\prime}}, q\right)$. Before we apply an iterative procedure to transform $\mu_{0}^{\prime}$ into a stable matching, we first establish a few properties of $\mu_{0}^{\prime}$.

$\mathbf{P 1}\left(\boldsymbol{\mu}_{\mathbf{0}}^{\prime}\right) \quad \mu_{0}^{\prime}$ is individually rational with respect to $\left(Q_{W^{\prime}}, P_{-W^{\prime}}, q\right)$.

Proof. Let $w \in W^{\prime}$. Since each $f \in \mu_{0}^{\prime}(w)=I^{\mu}(w)$ is acceptable for $w$ with respect to $P_{w}$, it is also acceptable with respect to $Q_{w}$. Let $w \in W \backslash W^{\prime}$. Then, $\mu_{0}^{\prime}(w)=\mu(w)$. Since each $f \in \mu(w)$ is acceptable for $w$ with respect to $P_{w}$, each $f \in \mu_{0}^{\prime}(w)$ is also acceptable for $w$ with respect to $P_{w}$. Let $f \in F$. Then, by definition of $\mu_{0}^{\prime}, \mu_{0}^{\prime}(f) \subseteq \mu(f)$. Since each $w \in \mu(f)$ is acceptable for $f$ with respect to $P_{f}$, each $w \in \mu_{0}^{\prime}(f)$ is also acceptable for $f$ with respect to $P_{f}$. Hence, $\mu_{0}^{\prime}$ is individually rational with respect to $\left(Q_{W^{\prime}}, P_{-W^{\prime}}, q\right)$.

$\mathbf{P 2}\left(\boldsymbol{\mu}_{\mathbf{0}}^{\prime}\right)$ If $(w, f)$ is a blocking pair for $\mu_{0}^{\prime}$ with respect to $\left(Q_{W^{\prime}}, P_{-W^{\prime}}, q\right)$, then $w \notin W^{\prime}$.

Proof. Suppose $w \in W^{\prime}$. By (b1) in the definition of blocking pair, $f \notin \mu_{0}^{\prime}(w)$. Recall that $Q_{w}$ is a dropping strategy for which the firms in $\mu_{0}^{\prime}(w)$ are the only acceptable ones. This gives a contradiction to (b2) in the definition of blocking pair and the individual rationality of $\mu_{0}^{\prime}$ with respect to $\left(Q_{W^{\prime}}, P_{-W^{\prime}}, q\right)$, which was established in $\mathrm{P} 1\left(\mu_{0}^{\prime}\right)$. Hence, $w \notin W^{\prime}$. 
$\mathbf{P 3}\left(\boldsymbol{\mu}_{\mathbf{0}}^{\prime}\right)$ If $(w, f)$ is a blocking pair for $\mu_{0}^{\prime}$ with respect to $\left(Q_{W^{\prime}}, P_{-W^{\prime}}, q\right)$, then $\left|\mu_{0}^{\prime}(f)\right|<q_{f}$.

Proof. Suppose it is not the case. Then, $\left|\mu_{0}^{\prime}(f)\right|=q_{f}$. Since $\mu_{0}^{\prime}(f) \subseteq \mu(f)$ and $|\mu(f)| \leq q_{f}$, $\mu_{0}^{\prime}(f)=\mu(f)$. By P2 $\left(\mu_{0}^{\prime}\right), w \notin W^{\prime}$. Hence, $\mu_{0}^{\prime}(w)=\mu(w)$. So, $(w, f)$ also blocks $\mu$ with respect to $\left(P_{W^{\prime}}^{\prime}, P_{-W^{\prime}}, q\right)$, which contradicts the stability of $\mu$ with respect to $\left(P_{W^{\prime}}^{\prime}, P_{-W^{\prime}}, q\right)$.

Set $\mu^{\prime}:=\mu_{0}^{\prime}$. As long as $\mu^{\prime}$ is not stable with respect to $\left(Q_{W^{\prime}}, P_{-W^{\prime}}, q\right)$, apply the following procedure.

\section{Begin Procedure.}

By $\mathrm{P} 1\left(\mu^{\prime}\right)$, there is at least one blocking pair for $\mu^{\prime}$ with respect to $\left(Q_{W^{\prime}}, P_{-W^{\prime}}, q\right)$. Let $f^{\prime}$ be a firm that is a member of one such blocking pair. Among all workers $w$ involved in blocking pairs $\left(w, f^{\prime}\right)$ for $\mu^{\prime}$ with respect to $\left(Q_{W^{\prime}}, P_{-W^{\prime}}, q\right)$, let $w^{\prime}$ be the most preferred worker with respect to $P_{f^{\prime}}$. By $\mathrm{P} 2\left(\mu^{\prime}\right), w^{\prime} \notin W^{\prime}$. By P3 $\left(\mu^{\prime}\right),\left|\mu^{\prime}\left(f^{\prime}\right)\right|<q_{f^{\prime}}$. Define

$$
\mu^{\prime \prime}(w)= \begin{cases}\mu^{\prime}\left(w^{\prime}\right) \cup f^{\prime} & \text { if } w=w^{\prime} \text { and }\left|\mu^{\prime}\left(w^{\prime}\right)\right|<q_{w^{\prime}} \\ \left(\mu^{\prime}\left(w^{\prime}\right) \cup f^{\prime}\right) \backslash \arg \min _{P_{w^{\prime}}}\left\{f: f \in \mu^{\prime}\left(w^{\prime}\right)\right\} & \text { if } w=w^{\prime} \text { and }\left|\mu^{\prime}\left(w^{\prime}\right)\right|=q_{w^{\prime}} \\ \mu^{\prime}(w) & \text { if } w \in W \backslash\left\{w^{\prime}\right\} .\end{cases}
$$

Then,

$\mathbf{P} 1\left(\boldsymbol{\mu}^{\prime \prime}\right) \mu^{\prime \prime}$ is individually rational with respect to $\left(Q_{W^{\prime}}, P_{-W^{\prime}}, q\right)$;

$\mathbf{P 2}\left(\boldsymbol{\mu}^{\prime \prime}\right)$ If $(w, f)$ is a blocking pair for $\mu^{\prime \prime}$ with respect to $\left(Q_{W^{\prime}}, P_{-W^{\prime}}, q\right)$, then $w \notin W^{\prime}$; and

$\mathbf{P 3}\left(\boldsymbol{\mu}^{\prime \prime}\right)$ If $(w, f)$ is a blocking pair for $\mu^{\prime \prime}$ with respect to $\left(Q_{W^{\prime}}, P_{-W^{\prime}}, q\right)$, then $\left|\mu^{\prime \prime}(f)\right|<q_{f}$.

Set $\mu^{\prime}:=\mu^{\prime \prime}$.

\section{End Procedure.}

In each iteration, one worker $w^{\prime} \notin W^{\prime}$ gets a strictly better match (with respect to $P_{w^{\prime}}$ ) and all other workers keep their match. (This follows from the fact that firm $f^{\prime}$ has a vacant position in $\mu^{\prime}$.) Therefore, the iterative procedure terminates after a finite number of steps. The resulting matching $\mu^{*}$ is stable with respect to $\left(Q_{W^{\prime}}, P_{-W^{\prime}}, q\right)$. Let $w \in W^{\prime}$. Since in each iteration of the procedure $w$ keeps it match, $\mu^{*}(w)=\mu_{0}^{\prime}(w)=I^{\mu}(w)$. Note that in $\mu^{*}$ agent $w$ is assigned to all its acceptable partners (with respect to $Q_{w}$ ). Hence, by Alkan (2002, Proposition 6), for each stable matching $\nu \in S\left(Q_{W^{\prime}}, P_{-W^{\prime}}, q\right), \nu(w)=\mu^{\star}(w)=I^{\mu}(w)$. By stability of $\varphi, \varphi_{w}\left(Q_{W^{\prime}}, P_{-W^{\prime}}, q\right)=I^{\mu}(w)$, which we needed to establish.

It only remains to show that in each iteration, $\mu^{\prime \prime}$ is a matching that satisfies $\mathrm{P} 1\left(\mu^{\prime \prime}\right), \mathrm{P} 2\left(\mu^{\prime \prime}\right)$, and P3 $\left(\mu^{\prime \prime}\right)$. We do this by induction. Suppose that in iteration 1 up to $k-1$ the resulting matching satisfies P1(.), P2(.), and P3(.). Let $\mu^{\prime}$ be the matching at the beginning of iteration $k$ (and suppose it is not stable with respect to $\left(Q_{W^{\prime}}, P_{-W^{\prime}}, q\right)$ ). (Hence, $\mathrm{P} 1\left(\mu^{\prime}\right), \mathrm{P} 2\left(\mu^{\prime}\right)$, and $\mathrm{P} 3\left(\mu^{\prime}\right)$ hold.) We will show that the matching $\mu^{\prime \prime}$ that is obtained in iteration $k$ satisfies $\mathrm{P} 1\left(\mu^{\prime \prime}\right)$, $\mathrm{P} 2\left(\mu^{\prime \prime}\right)$, and $\mathrm{P} 3\left(\mu^{\prime \prime}\right)$.

Proof of $\mathrm{P} 1\left(\mu^{\prime \prime}\right)$. By the induction hypothesis, $\mu^{\prime}$ is individually rational with respect to $\left(Q_{W^{\prime}}, P_{-W^{\prime}}, q\right)$. The only new mates in $\mu^{\prime \prime}$ with respect to $\mu^{\prime}$ are the pair $\left\{w^{\prime}, f^{\prime}\right\}$. Since $\left(w^{\prime}, f^{\prime}\right)$ is a blocking pair for $\mu^{\prime}$ and since $\mu^{\prime}$ is individually rational with respect to $\left(Q_{W^{\prime}}, P_{-W^{\prime}}, q\right)$, it 
immediately follows that $w^{\prime}$ and $f^{\prime}$ are mutually acceptable with respect to $\left(Q_{W^{\prime}}, P_{-W^{\prime}}, q\right)$. Therefore, $\mu^{\prime \prime}$ is individually rational with respect to $\left(Q_{W^{\prime}}, P_{-W^{\prime}}, q\right)$.

$\diamond$

Proof of P2 $\left(\mu^{\prime \prime}\right)$. Suppose $w \in W^{\prime}$. Since $(w, f)$ blocks $\mu^{\prime \prime}$ with respect to $\left(Q_{W^{\prime}}, P_{-W^{\prime}}, q\right)$, $f \notin \mu^{\prime \prime}(w)$. By the induction hypothesis, in iterations 1 up to $k$, agent $w$ has kept it original match, i.e., $\mu^{\prime \prime}(w)=\mu_{0}^{\prime}(w)$. Hence, $w$ blocks $\mu^{\prime \prime}$ together with $f \notin \mu_{0}^{\prime}(w)$. Recall that $Q_{w}$ is a dropping strategy for which the firms in $\mu_{0}^{\prime}(w)$ are the only acceptable ones for $w$. This gives a contradiction to (b2) in the definition of blocking pair and the individual rationality of $\mu_{0}^{\prime}$ with respect to $\left(Q_{W^{\prime}}, P_{-W^{\prime}}, q\right)$, which was established in $\mathrm{P} 1\left(\mu_{0}^{\prime}\right)$. Hence, $w \notin W^{\prime}$.

Proof of P3 $\left(\mu^{\prime \prime}\right)$. Let $(w, f)$ be a blocking pair for $\mu^{\prime \prime}$ with respect to $\left(Q_{W^{\prime}}, P_{-W^{\prime}}, q\right)$. By $\mathrm{P} 2\left(\mu^{\prime \prime}\right), w \notin W^{\prime}$. Suppose $\left|\mu^{\prime \prime}(f)\right|=q_{f}$. Then, by (b3) in the definition of blocking pair, $w P_{f} \tilde{w}$ for some $\tilde{w} \in \mu^{\prime \prime}(f)$. We distinguish between two cases.

Case I. $(\tilde{w}, f)$ was a blocking pair matched in some iteration $l, l \leq k$.

By the induction hypothesis, in iterations $l+1$ up to $k$, worker $w \notin W^{\prime}$ either keeps its match from iteration $l$ or obtains a strictly better match by (possibly repeatedly) adding an acceptable firm and/or replacing its least preferred mate by a more preferred firm (if its quota is reached). Therefore, since $(w, f)$ is a blocking pair for $\mu^{\prime \prime}$ at the end of iteration $k, w$ is also willing to block (with $f$ ) the initial matching in iteration $l$ and $w$ and $f$ are not mates at the initial matching in iteration $l$. Since $w P_{f} \tilde{w}$, firm $f$ did not block with the best possible worker in iteration $l$, which contradicts the definition of the procedure.

Case II. $\tilde{w}$ is matched with $f$ in all matchings of iterations $1, \ldots, k$.

By the induction hypothesis, in iterations 1 up to $k$, worker $w \notin W^{\prime}$ either keeps its match $\mu_{0}^{\prime}(w)$ or obtains a strictly better match by (possibly repeatedly) adding an acceptable firm and/or replacing its least preferred mate by a more preferred firm (if its quota is reached). Therefore, since $(w, f)$ is a blocking pair for $\mu^{\prime \prime}$ at the end of iteration $k, w$ is also willing to block (with $f$ ) matching $\mu_{0}^{\prime}$ (with respect to $P_{w}$ ) and $w \notin \mu_{0}^{\prime}(f)$. Since $w P_{f} \tilde{w}$ and (by assumption) $\tilde{w} \in \mu_{0}^{\prime}(f)$, $(w, f)$ is a blocking pair for $\mu_{0}^{\prime}$ with respect to $\left(P_{W^{\prime}}^{\prime}, P_{-W^{\prime}}, q\right)$. Since $w \notin W^{\prime}$, it follows from the definition of $\mu_{0}^{\prime}$ that $\mu(\tilde{w})=\mu_{0}^{\prime}(\tilde{w})$. Hence, $(w, f)$ is a blocking pair for $\mu$ with respect to $\left(P_{W^{\prime}}^{\prime}, P_{-W^{\prime}}, q\right)$, which contradicts the stability of $\mu=\varphi\left(P_{W^{\prime}}^{\prime}, P_{-W^{\prime}}, q\right)$.

\begin{tabular}{|c|c|c|c|c|c|c|c|}
\hline & \multicolumn{3}{|c|}{ Quotas } & \multirow{2}{*}{$\begin{array}{c}\varphi \text {-exhaustive } \\
\text { for } \\
\text { worker } w\end{array}$} & \multicolumn{2}{|c|}{ Quotas } & \multirow{2}{*}{$\begin{array}{c}\varphi \text {-exhaustive } \\
\text { for } \\
\text { a group of workers }\end{array}$} \\
\hline & $\begin{array}{c}\text { Worker } \\
w\end{array}$ & $\begin{array}{c}\text { Other } \\
\text { workers }\end{array}$ & Firms & & Workers & Firms & \\
\hline \multirow{2}{*}{$\begin{array}{l}\text { Truncation } \\
\text { correspondence }\end{array}$} & $=1$ & $\geq 1$ & $\geq 1$ & $+($ Theorem 1$)$ & \multirow[b]{2}{*}{$=1$} & \multirow[b]{2}{*}{$=1$} & \multirow[b]{2}{*}{ - (Example 1) } \\
\hline & $>1$ & $=1$ & $=1$ & - (Example 2) & & & \\
\hline $\begin{array}{l}\text { Dropping } \\
\text { correspondence }\end{array}$ & \multicolumn{2}{|c|}{$\geq 1$} & $\geq 1$ & $+($ Theorem 2$)$ & $\geq 1$ & $\geq 1$ & $+($ Theorem 2$)$ \\
\hline
\end{tabular}

Table 5: Summary of results. Given the quotas of the workers and firms, + (-) means that the correspondence is (not necessarily) exhaustive.

We conclude with Table 5, which summarizes all our (positive and negative) findings. 


\section{References}

[1] A. Alkan (1999). On the Properties of Stable Many-to-Many Matchings under Responsive Preferences. In: Alkan, A., Aliprantis, C.D., and N.C. Yannelis (Eds.), Current Trends in Economics: Theory and Applications. Vol. 8. Studies in Economic Theory. Berlin, Heidelberg: Springer.

[2] A. Alkan (2001). On Preferences over Subsets and the Lattice Structure of Stable Matchings. Review of Economic Design 6(1): 99-111.

[3] A. Alkan (2002). A Class of Multipartner Matching Markets with a Strong Lattice Structure. Economic Theory 19(4): 737-746.

[4] M. Baïou and M. Balinski (2000). Many-to-Many Matchings: Stable Polyandrous Polygamy (or Polygamous Polyandry). Discrete Applied Mathematics 101(1): 1-12.

[5] C. Blair (1988). The Lattice Structure of the Set of Stable Matchings with Multiple Partners. Mathematics of Operations Research 13(4): 619-628.

[6] P. Coles (2009). Optimal Truncation in Matching Markets. Mimeo. Harvard Business School.

[7] L.E. Dubins and D.A. Freedman (1981). Machiavelli and the Gale-Shapley Algorithm. American Mathematical Monthly 88(7): 485-494.

[8] F. Echenique and J. Oviedo (2006). A Theory of Stability in Many-to-Many Matching Markets. Theoretical Economics 1(2): 233-273.

[9] L. Ehlers (2008). Truncation Strategies in Matching Markets. Mathematics of Operations Research 33(2): 327-335.

[10] L. Fleiner (2003). A Fixed-Point Approach to Stable Matchings and Some Applications. Mathematics of Operations Research 28(1): 103-126.

[11] D. Gale and L.S. Shapley (1962). College Admissions and the Stability of Marriage. American Mathematical Monthly 69(1): 9-15.

[12] A.S. Kelso and V.P. Crawford (1982). Job Matching, Coalition Formation, and Gross Substitutes. Econometrica 50(6): 1483-1504.

[13] B. Klaus and M. Walzl (2009). Stable Many-to-Many Matchings with Contracts. Journal of Mathematical Economics, 45(7): 422-434.

[14] F. Klijn and A. Yazıcı (2011). A Many-to-Many 'Rural Hospital Theorem'. Mimeo. Department of Economics, University of Rochester. 
[15] F. Kojima and P.A. Pathak (2009). Incentives and Stability in Large Two-Sided Matching Markets. American Economic Review, 99(3): 608-627.

[16] H. Konishi and M.U. Ünver (2006). Credible Group-Stability in Many-to-Many Matching Problems. Journal of Economic Theory, 129(1): 57-80.

[17] J. Ma (2010). The Singleton Core in the College-admissions Problem and its Application to the National Resident Matching Program (NRMP). Games and Economic Behavior, 69(1): $150-164$.

[18] R. Martínez, J. Massó, A. Neme, and J. Oviedo (2004). An Algorithm to Compute the Set of Many-to-Many Stable Matchings. Mathematical Social Sciences 47(2): 187-210.

[19] S. Mongell and A.E. Roth (1991). Sorority Rush as a Two-Sided Matching Mechanism. American Economic Review 81(3): 441-464.

[20] A.E. Roth (1982). The Economics of Matching: Stability and Incentives. Mathematics of Operations Research 7(4): 617-628.

[21] A.E. Roth (1984a). Stability and Polarization of Interests in Job Matching. Econometrica $52(1): 47-58$.

[22] A.E. Roth (1984b). The Evolution of the Labor Market for Medical Interns and Residents: A Case Study in Game Theory. Journal of Political Economy 92(6): 991-1016.

[23] A.E. Roth (1985a). The College Admission Problem is not Equivalent to the Marriage Problem. Journal of Economic Theory 36(2): 277-288.

[24] A.E. Roth (1985b). Conflict and Coincidence of Interest in Job Matching: Some New Results and Open Questions. Mathematics of Operations Research 10(3): 379-389.

[25] A.E. Roth (1991). A Natural Experiment in the Organization of Entry-Level Labor Markets: Regional Markets for New Physicians and Surgeons in the United Kingdom. American Economic Review 81(3), 415-440.

[26] A.E. Roth and U.G. Rothblum (1999). Truncation Strategies in Matching Markets - In Search of Advice for Participants. Econometrica 67(1): 21-43.

[27] A.E. Roth and M.A.O. Sotomayor (1989). The College Admissions Problem Revisited. Econometrica 57(3): 559-570.

[28] A.E. Roth and M.A.O. Sotomayor (1990). Two-Sided Matching: A Study in GameTheoretic Modeling and Analysis. Econometric Society Monograph Series. New York: Cambridge University Press.

[29] A.E. Roth and J.H. Vande Vate (1991). Incentives in Two-Sided Matching with Random Stable Mechanisms. Economic Theory 1(1): 31-44. 
[30] T. Sönmez (1997). Manipulation via Capacities in Two-Sided Matching Markets. Journal of Economic Theory 77(1): 197-204.

[31] M.A.O. Sotomayor (1999a). The Lattice Structure of the Set of Stable Outcomes of the Multiple Partners Assignment Game. International Journal of Game Theory 28(4): 567583.

[32] M.A.O. Sotomayor (1999b). Three Remarks on the Many-to-Many Stable Matching Problem. Mathematical Social Sciences 38(1): 55-70.

[33] M.A.O. Sotomayor (2004). Implementation in the Many-to-Many Matching Market. Games and Economic Behavior 46(1): 199-212. 